\title{
Importancia de las competencias directivas desde la percepción
}

\section{de los empresarios arequipeños}

\section{Laura Chávez Cáceres, Walter L. Arias Gallegos}

\author{
Departamento de Administración de Negocios \\ Universidad Católica San Pablo, Arequipa \\ Departamento de Psicología \\ Universidad Católica San Pablo, Arequipa
}

Esta investigación tiene como objetivo determinar cuál es la percepción de los empresarios arequipeños con respecto a la importancia que asignan a las competencias directivas según el modelo empresarial antropológico planteado por Juan Antonio Pérez López (1993). Para la recolección de datos, se utilizó un cuestionario diseñado por profesores del IESE Business School (España), que fue adaptado para la investigación con adecuados índices de validez y confiabilidad, y aplicado individualmente a los directivos de 70 empresas de 3 rubros diferentes. Entre los hallazgos, se observó que las competencias directivas más valoradas fueron honestidad, orientación al cliente, autocontrol, coaching, visión de negocio, autoconocimiento, concentración, trabajo en equipo, gestión de recursos, gestión del tiempo, networking, delegación, equidad, autocritica y aprendizaje personal.

Palabras clave: competencias directivas, empresarios arequipeños, administración de negocios, capital social.

\section{Relevance of managerial skills since Arequipenian manager's perception}

This research aims to determine which is the perception of Arequipenian managers about the importance assigned to the management skills according to the anthropological model proposed by Juan Antonio Pérez López (1993). For data collection, a questionnaire was used, designed by professors of the IESE Business School (Spain), which was adapted for the investigation with good levels of validity and reliability, and was applied in an individual way in 70 companies. The main findings were that the most valued management skills, were honesty, customer focus, self-control, coaching, business insight, self-knowledge, concentration, teamwork, resource management, time management, networking, delegation, equity, self-criticism and personal learning.

Keywords: Managerial skills, Arequipenian managers, business administration, social capital.

\section{Importância das competências diretivas desde a percepção dos empresários de Arequipa}

A seguinte pesquisa teve como objetivo determinar a percepçáo dos empresários da cidade de Arequipa (Peru) respeito da importância que dão às competências diretivas segundo o modelo empresarial antropológico de Juan Antonio Pérez López (1993). Para a recoleição de dados foi empregado um instrumento feito pela IESE Business School (Espanha), adaptado para o estudo e com níveis suficientes de validez e confiabilidade. O instrumento foi respondido individualmente por diretivos de 70 empresas. Os resultados principais confiram que as competências diretivas mais valoradas são honestidade, orientação ao cliente, autocontrol, coaching, visão de negócio, autoconhecimento, concentração, trabalho em equipes, gestão de recursos, gestão do tempo, networking, delegação, equidade, autocrítica e aprendizagem personal.

Palavras chave: competências diretivas, empresários, Arequipa, administração de negocios, capital social. 


\section{Introducción}

La dinámica económica que vive el país y su apertura al mundo exigen la presencia de nuevos profesionales en gestión, con mayor competitividad técnica y mayores habilidades en management. Esta realidad va de la mano con una mayor especialización de los perfiles de puesto a distintos niveles, habiéndose perfeccionado y afinado también los sistemas de selección y de gestión del talento humano (Alles, 2012) o de los llamados recursos humanos en las organizaciones (Chiavenato, 2002). En ese marco, esta investigación tiene como finalidad determinar cuál es la percepción de los empresarios arequipeńos con respecto a la importancia que les asignan a las competencias directivas según el modelo antropológico de Juan Antonio Pérez López (1993) en tres rubros de empresa: servicios, industria y comercio.

Se decidió evaluar la percepción, debido a que se pretende determinar cuál es la apreciación que el empresario arequipeño tiene acerca de las competencias directivas, definidas como "comportamientos habituales y observables que un estudiante, egresado o profesional debería tener para lograr un exitoso desempeño en su función directiva» (Chávez, 2013, p. 20). En ese sentido, se optó por indagar acerca de la importancia que dichas competencias tienen para los empresarios arequipeños con el fin de identificar cuáles son las competencias directivas que realmente valoran.

De acuerdo con Quiñones (2013), el talento se ha convertido en un recurso escaso en la economía mundial, puesto que actúa como un filtro de otros factores implicados en la producción. Es decir, a través del talento humano, se mediatizan una amplia diversidad de posibilidades con las que cuenta la empresa. Ello supone que es necesario contar con administradores y gerentes con competencias directivas, capaces de gestionar su propio talento y el de los demás. Sin embargo, a pesar de que la carrea de Administración se encuentra sobrepoblada en el Perú (el 53\% de los estudiantes universitarios corresponde a carreras de Derecho, Administración y otras Ciencias Sociales), los puestos más difíciles de cubrir en las empresas peruanas corresponden a los de personal directivo y administrativo (Yamada, Castro y Asmat, 2013).

Asimismo, en los escenarios laborales, se está poniendo especial énfasis en las competencias de eficacia y eficiencia personal, $y$, de manera muy especial, en las emociones positivas en el trabajo (Salanova, Martínez y Llorens, 2005) y el civismo organizacional (Rodríguez, Martínez y Salanova, 2014). Esto responde a que ambos aspectos impactan favorablemente en la productividad y la rentabilidad de las empresas (Fischman, 2010), así como en el bienestar de los trabajadores (Salanova, 2008).

De este modo, nos encontramos en la era del conocimiento y el talento humano, en la que el capital social tiene mayor relevancia que los recursos económicos y las personas no son vistas como meros recursos, sino como la principal fuente de desarrollo organizacional (Robbins, 1999). Así, la diferencia entre una empresa y otra ya no es el capital o la tecnología con la que cuenta, sino la manera como gestiona el talento de sus trabajadores (Chiavenato, 2009). De este modo, el capital humano es un recurso estratégico que le otorga ventajas competitivas a las empresas que los gestionan eficaz y eficientemente.

Puede decirse, también, que los modelos mecanicistas que abogaban por los beneficios económicos (Friedman y Friedman, 1986) o la organización eficiente del trabajo (Taylor, 1973) han sido sustituidos por modelos psicosociológicos y antropológicos de empresa. En los primeros, si bien hay una focalización en la persona, todavía se le considera como un recurso, y se enfatiza en 
los aspectos directivos y funcionales del trabajo (Fayol, 1973). Asimismo, se valoran y redimensionan los aspectos estratégicos de la administración de los negocios (Drucker, 2003) orientándolos hacia la promoción del bienestar y la salud del trabajador, pues, si este se encuentra satisfecho, su productividad será mayor.

Mientras, en los modelos antropológicos, se entiende a la empresa como una realidad humana y se parte de tres valores fundamentales: i) una buena relación entre lo producido y lo consumido por la empresa, ii) un grado de satisfacción actual con aquellos que se relacionan con la empresa (stakeholders) y iii) una contribución al desarrollo personal de quienes colaboran con ella (Pérez López, 1993). Por esta razón, como señalan Loza y Habisch (2009), una empresa cimentada en una antropología verdaderamente humana se anticipa al concepto de responsabilidad social corporativa o empresarial, puesto que la responsabilidad social empresarial no está sujeta al mandato legal ni se lleva a cabo como mera filantropía, sino que emana de la auténtica convicción de obrar rectamente (Barroso 2008). A partir de ello, la visión antropológica se encuentra indisolublemente vinculada con la ética en los negocios (Manzone 2007) y ha merecido diversos análisis de la coyuntura generada por la crisis económica global de hace unos años atrás (Montoya, 2010).

Asimismo, de acuerdo con el modelo antropológico, se pueden distinguir tres tipos de motivación en los colaboradores: la extrínseca, que depende de las recompensas y los castigos que recibe el trabajador; la intrínseca, que se relaciona con la satisfacción y la autorrealización del trabajador; y la trascendente, que se vincula con la satisfacción de las necesidades de otras personas. Estos tres tipos de motivación se encuentran impulsados por necesidades materiales, cognitivas y afectivas. A su vez, determinan distintos valores en la organización, como son la eficacia, la atractividad y la unidad (Ferreiro y Alcázar, 2002).

Figura 1. Competencias y valores del modelo antropológico propuesto

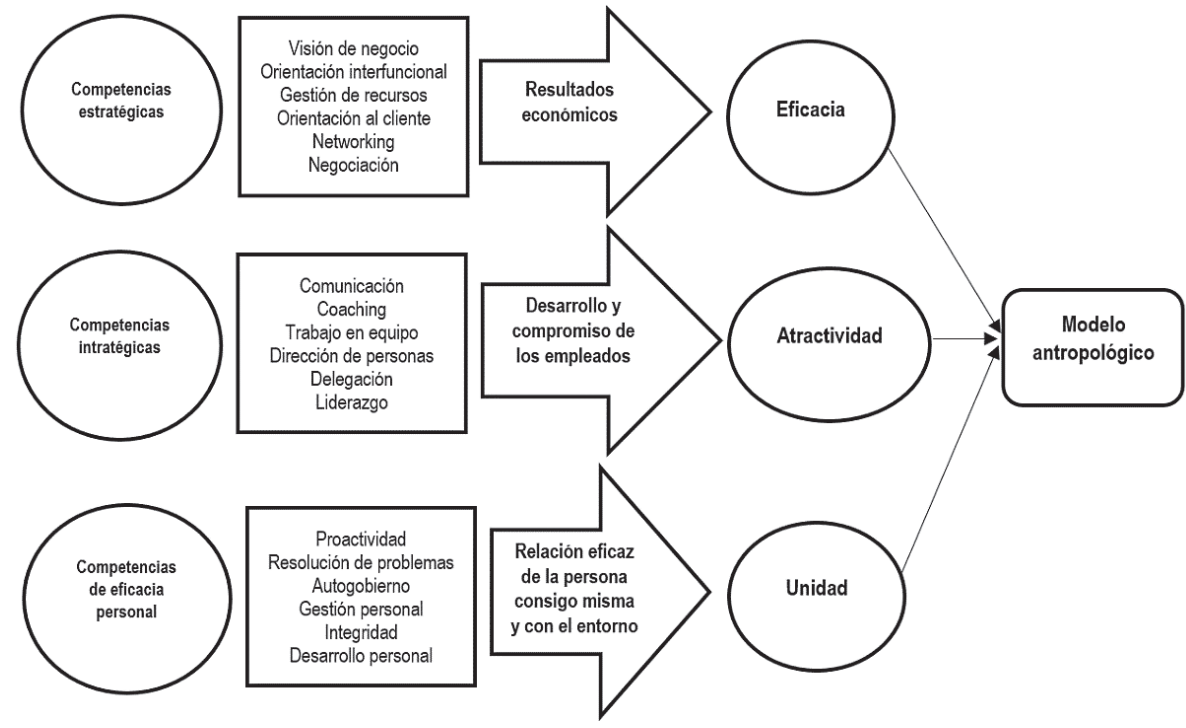

Fuente: Pérez López (1993). 
La eficacia es la medida en que una organización es capaz de conseguir la adhesión de los individuos a través de la satisfacción de las motivaciones extrínsecas. La atractividad, por su parte, es la medida en que los individuos se adhieren a la organización no por lo que reciben, sino por la satisfacción de necesidades intrínsecas y trascendentes. En cuanto a la unidad, esta es la medida en que la adhesión a la organización está específicamente vinculada con la motivación trascendente (Pérez López, 1993).

En un estudio previo, realizado en la ciudad de Arequipa sobre la base de estos supuestos empresariales y antropológicos, se evaluó a un grupo de trabajadores de una agencia bancaria de la localidad. Dicha evaluación reportó que ciertos componentes de la gratitud, como son la reciprocidad y la cualidad sentimental, tienen un efecto positivo y significativo sobre la motivación trascendente, datos que son consistentes con el modelo antropológico propuesto (Menautt, Checa y Arias, 2016).

En relación con las competencias, las directivas estratégicas se alinean con las necesidades materiales, los motivos extrínsecos y los valores de eficacia, mientras que las competencias directivas intratégicas, con las necesidades cognitivas, los motivos intrínsecos y los valores de atractividad. Finalmente, las competencias directivas de eficacia personal se alinean con las necesidades afectivas, los motivos trascendentes y los valores de unidad en la organización (Covey, 1993).

$\mathrm{Al}$ respecto, cabe anotar que existen diversas propuestas teóricas sobre las competencias que las definen como un listado de comportamientos que ciertas personas poseen más que otras, lo que las hace más eficaces para una situación dada (Alles 2010). Asimismo, existen diversas tipologías que las diferencian de las competencias teóricas, prácticas, sociales y de conocimiento
(Jolis 1998). Sin embargo, en contraste con este enfoque, nosotros nos basamos en la propuesta teórica de Cardona, Chinchilla y García-Lombardía (2001), quienes las dividen entre competencias técnicas y competencias directivas. Las primeras se refieren a aquellos atributos o rasgos distintivos que requiere un trabajador en un puesto de trabajo determinado, mientras que las segundas consisten en aquellos comportamientos observables y habituales que justifican el éxito de una persona en su función directiva.

Ahora bien, una clasificación de las competencias directivas planteada por Pérez López (1993) las categoriza en tres niveles dentro de un modelo antropológico de empresa: estratégicas, intratégicas y de eficacia personal. Las competencias directivas estratégicas son las que generan valor económico para la empresa, las intratégicas se enfocan en el desarrollo de los colaboradores y las de eficacia personal constituyen aquellas que facilitan una relación adecuada de la persona para con el entorno laboral (Covey 1993). Cabe resaltar que es muy importante que las tres dimensiones se vinculen entre sí, porque actúan complementariamente.

En la Tabla 1, se sistematizan las diferentes competencias directivas en función de su tipo. Así, las competencias directivas estratégicas son seis. La visión de negocio implica reconocer y aprovechar las oportunidades, los peligros y las fuerzas externas que repercuten en la competitividad del negocio. La orientación interfuncional supone mirar más allá de los límites de la función hasta abarcar el impacto de las propias actuaciones en otras unidades de la empresa desarrollando la cooperación. La gestión de recursos consiste en utilizar los recursos del modo más idóneo, rápido, económico y eficaz para obtener los resultados deseados. La orientación al cliente, como su nombre lo indica, supone responder con prontitud y eficacia a las sugerencias y necesidades del cliente. El networking 
implica que el colaborador desarrolle y mantenga una amplia red de relaciones con personas clave dentro de la empresa y del sector, mientras que la negociación consiste en llegar a acuerdos que satisfacen los intereses de ambas partes.

Las competencias directivas intratégicas son también seis. La comunicación es escucha y transmisión de ideas de manera efectiva, a través de procedimientos formales e informales, y proporcionando datos concretos para respaldar sus observaciones y conclusiones. La dirección con las personas refiere a la asignación de objetivos y tareas a las personas adecuadas para realizar el trabajo y planificar su seguimiento. La delegación se manifiesta a partir de la preocupación de que los integrantes del equipo de trabajo dispongan de la capacidad para tomar decisiones y de los recursos necesarios para lograr sus objetivos. Por su parte, el coaching permite ayudar a los colaboradores a descubrir sus áreas de mejora, y a desarrollar sus habilidades y capacidades profesionales. El trabajo en equipo fomenta un ambiente de colaboración, comunicación y confianza entre los miembros del equipo y los estimula hacia el logro de los objetivos comunes. Finalmente, el liderazgo da sentido al trabajo de sus colaboradores animando, ilusionando y motivándolos a conseguir los objetivos que se proponen.

En cuanto a las competencias directivas de eficacia personal, estas se componen de seis factores con sus respectivas dimensiones. La proactividad, por ejemplo, engloba la iniciativa, la creatividad y el optimismo. La solución de problemas comprende la intuición, la capacidad de análisis y la toma de decisiones, mientras que el autogobierno abarca la concentración, la tenacidad y el autocontrol. La gestión personal se compone de la gestión del tiempo, la gestión del estrés y la gestión de la incertidumbre. La integridad, por su parte, comprende la credibilidad, la honestidad y la equidad.
Por último, el desarrollo personal se compone de la autocrítica, el autoconocimiento y el aprendizaje personal.

\section{Tabla 1. Competencias directivas}

\begin{tabular}{|c|c|c|}
\hline \multirow{7}{*}{$\begin{array}{l}\text { Competencias } \\
\text { estratégicas }\end{array}$} & \multicolumn{2}{|c|}{ Competencias directivas } \\
\hline & \multicolumn{2}{|c|}{ Visión de negocio } \\
\hline & \multicolumn{2}{|c|}{ Orientación interfuncional } \\
\hline & \multicolumn{2}{|c|}{ Gestión de recursos } \\
\hline & \multicolumn{2}{|c|}{ Orientación al cliente } \\
\hline & \multicolumn{2}{|l|}{ Networking } \\
\hline & \multicolumn{2}{|l|}{ Negociación } \\
\hline \multirow{6}{*}{$\begin{array}{l}\text { Competencias } \\
\text { intratégicas }\end{array}$} & \multicolumn{2}{|l|}{ Comunicación } \\
\hline & \multicolumn{2}{|l|}{ Coaching } \\
\hline & \multicolumn{2}{|c|}{ Trabajo en equipo } \\
\hline & \multicolumn{2}{|c|}{ Dirección de personas } \\
\hline & \multicolumn{2}{|l|}{ Delegación } \\
\hline & \multicolumn{2}{|l|}{ Liderazgo } \\
\hline \multirow{18}{*}{$\begin{array}{l}\text { Competencias } \\
\text { de eficacia } \\
\text { personal }\end{array}$} & \multirow[t]{3}{*}{ Proactividad } & Iniciativa \\
\hline & & Creatividad \\
\hline & & Optimismo \\
\hline & \multirow{3}{*}{$\begin{array}{l}\text { Resolución } \\
\text { de problemas }\end{array}$} & Intuición \\
\hline & & Capacidad de análisis \\
\hline & & Toma de decisiones \\
\hline & \multirow{3}{*}{$\begin{array}{l}\text { Autogo- } \\
\text { bierno }\end{array}$} & Concentración \\
\hline & & Tenacidad \\
\hline & & Autocontrol \\
\hline & \multirow{3}{*}{$\begin{array}{l}\text { Gestión } \\
\text { personal }\end{array}$} & Gestión de tiempo \\
\hline & & Gestión de estrés \\
\hline & & Gestión de incertidumbre \\
\hline & \multirow[t]{3}{*}{ Integridad } & Credibilidad \\
\hline & & Honestidad \\
\hline & & Equidad \\
\hline & \multirow{3}{*}{$\begin{array}{l}\text { Desarrollo } \\
\text { personal }\end{array}$} & Autocrítica \\
\hline & & Autoconocimiento \\
\hline & & Aprendizaje personal \\
\hline
\end{tabular}

Fuente: Cardona et al. (2001). 
La presente investigación pretende responder a la siguiente interrogante: ¿Cuál es la percepción de los empresarios arequipeños con respecto a la importancia que asignan a las competencias directivas según el modelo antropológico de empresa? Para ello, tomamos como modelo el estudio de Cardona et al. (2001), en el que se aplicó un cuestionario a 1147 empresas para identificar las competencias directivas más valoradas por los empresarios. De ese total, solo el 13\% respondió, es decir, 103 empresas españolas y 45 empresas de Europa y América. A partir de sus respuestas, se reportó que los empresarios buscan un equilibrio entre las competencias directivas estratégicas, intratégicas y de eficiencia personal, pero con una ligera preferencia por las competencias de desarrollo personal.

\section{Método}

La presente investigación tiene carácter exploratorio (Hernández, Fernández y Baptista, 2010), pues no se ha efectuado un estudio similar en la ciudad de Arequipa ni existe registro de ello a nivel nacional. Por tanto, no se han planteado una hipótesis de investigación.

\subsection{Muestra}

La muestra estuvo conformada por 70 empresas locales ubicadas en la ciudad de Arequipa, que fueron seleccionadas de manera no probabilística, según hayan brindado el acceso para la realización de la presente investigación. De las 70 organizaciones, el 53\% fueron empresas de servicios - tales como transporte, turismo, instituciones financieras, servicios públicos y privados_- el 25\% fueron empresas industriales, tanto manufactureras como extractivas y agropecuarias; y, finalmente, el 22\% restante lo constituyeron empresas comerciales, mayoristas minoristas y comisionistas.

\subsection{Instrumentos}

Se aplicó el cuestionario diseñado por Cardona et al. (2001) en la Escuela de Negocios IESE para valorar las competencias directivas en 148 empresas de todo el mundo. El cuestionario constó de 30 competencias conceptualizadas que se evaluaron en una escala tipo Likert que va de 1 («Muy mala») a 5 («Muy buena»), de acuerdo con la importancia asignada por el evaluador. En cuanto a las cualidades psicométricas del instrumento, se practicó un análisis de correlación ítem-test con la finalidad de valorar la homogeneidad de la prueba, mediante el cual se encontró que las correlaciones oscilaron entre 0,46 (para el ítem 3) y 0,79 para el ítem 18. Se concluyó, por tanto, que las escalas cumplen con el criterio de homogeneidad de los reactivos.

Para valorar la validez de constructo del instrumento, se aplicó, en primer lugar, la prueba Kaiser-MeyerOlkin, que obtuvo un valor de 0,769 para la escala de importancia, mientras que las medidas de adecuación muestral ofrecieron valores chi cuadrado $\left(x^{2}=1439,862\right.$; $\mathrm{gl}=435)$ altamente significativos $(\mathrm{p}<0,000)$. Todo ello indicó que la prueba puede ser factorizada. Se hallaron 7 factores, cuyo primer factor explicaba el 44,632\% de la varianza total. De este modo, los elevados porcentajes de varianza explicada, así como el hecho de que en los factores restantes solo saturaba un ítem, llevaron a considerarla como un instrumento unidimensional (Burga 2006). Asimismo, la confiabilidad se calculó mediante el método de consistencia interna a través de la prueba alfa de Cronbach, que ofreció un coeficiente de 0,947 . A partir de ello, puede decirse que el instrumento utilizado es válido y confiable.

\subsection{Procedimiento}

Para la aplicación de los instrumentos, se pactaron citas previas de coordinación con los directivos que laboran en las empresas seleccionadas. Primero, se realizó una 
presentación personal de la investigadora principal; luego, una presentación del tema en cuestión; y, finalmente, se procedió a explicar la metodología a emplear. Los directivos fueron evaluados en sus respectivas oficinas de manera individual y sin ningún tipo de interrupción durante un tiempo aproximado de 30 minutos por persona, incluida la explicación previa. Todos accedieron a participar de la investigación voluntariamente, y se les garantizó la reserva y la confidencialidad de la información personal y de los datos proporcionados.

\section{Resultados}

\subsection{Competencias directivas en las empresas de servicios}

Con respecto al rubro de servicios, se acudió a 37 empresas que conforman el 53\% del total de la muestra y se realizó una encuesta a cada una. Del total de entrevistados, fueron 7 mujeres $(18,9 \%)$ y 30 varones $(81,1 \%)$. En cuanto al tamaño de las empresas, el 40,54\% fueron grandes empresas; el 13,51\%, medianas empresas; el $27,03 \%$, pequeñas empresas; y el 18,93\%, microempresas. En cuanto al cargo de los empresarios, más del $50 \%$ de los entrevistados fueron jefes. Por otro lado, se logró entrevistar a 12 gerentes generales $(32,4 \%)$ y 6 gerentes de área (16,2\%). Con respecto a su profesión, predominó la carrera de Administración de Negocios (con un 56,76\%), seguida por la carrera de Contabilidad e Ingeniería Industrial, ambas con un 10,81\%.

En cuanto a la edad, se decidió categorizar esta variable, dada la variabilidad de resultados. La edad mínima fue de 22 años y la máxima de 56 años. Más de la mitad de entrevistados (el 51,35\%) se concentró en edades de 30 a 39 años, mientras que el menor porcentaje estuvo compuesto por los entrevistados entre 20 y 29 años. Finalmente, con porcentajes similares, se encontraron los entrevistados entre los rangos de 40 a 49 años, y 50 a 59 años. Sobre esta base, se puede decir que, a partir de los 30 años, se empieza a ocupar puestos directivos en mayor medida.

En cuanto a los valores descriptivos de la importancia de las competencias directivas, se observa que las ponderaciones son medias altas con desviaciones estándar bajas (Tabla 2). Como se puede apreciar en la Figura 2, en cuanto a la importancia de las competencias directivas en las empresas de servicios, los directivos asignaron puntuaciones altas a la mayoría de las competencias directivas. Las competencias que recibieron una mayor puntuación promedio fueron las de eficacia personal, específicamente, la honestidad. En segundo lugar, credibilidad, capacidad de análisis y la competencia intratégica de trabajo en equipo recibieron las mismas puntuaciones. Finalmente, se encuentran orientación al cliente y liderazgo.

Por otro lado, las competencias que recibieron las menores calificaciones promedio fueron autocrítica, autocontrol y networking. Luego, se evaluó cuáles son las diez competencias más importantes para los directivos. Las calificaciones promedio más elevadas, en su mayoría, se atribuyeron a las competencias de eficacia personal y las competencias intratégicas.

Asimismo, se practicó un análisis de correlación con la prueba Tau-b de Kendall entre los puntajes brutos de la importancia de las competencias directivas, con algunos datos ordinales reportados por los empresarios, como su edad, su cargo y el tamańo de la empresa. Se encontró así una correlación moderada negativa entre el cargo del evaluador y las competencias directivas -trabajo en equipo $(r=-, 304)$, honestidad $(r=-, 384)$ y aprendizaje personal $(\mathrm{r}=-, 304)-$, con un nivel de significancia menor de ,05. Ello indica que, mientras mayor es el cargo de los directivos en las empresas de servicios, estos asignan menos importancia a las competencias mencionadas.

Contabilidad y Negocios (13) 26, 2018 / ISSN 1992-1896 
Tabla 2. Valores descriptivos de la importancia de las competencias directivas en el rubro de servicios

\begin{tabular}{|c|c|c|c|c|c|c|c|}
\hline \multirow{7}{*}{$\begin{array}{l}\text { Competencias } \\
\text { estratégicas }\end{array}$} & & Rango & Mín. & Máx. & Media & D.E. & Varianza \\
\hline & Visión de negocio & 2 & 3 & 5 & 4,24 & 0,60 & 0,36 \\
\hline & Orientación interfuncional & 3 & 2 & 5 & 4,30 & 0,81 & 0,66 \\
\hline & Gestión de recursos & 2 & 3 & 5 & 4,36 & 0,68 & 0,47 \\
\hline & Orientación al cliente & 2 & 3 & 5 & 4,49 & 0,73 & 0,53 \\
\hline & Networking & 2 & 3 & 5 & 4,14 & 0,79 & 0,62 \\
\hline & Negociación & 2 & 3 & 5 & 4,27 & 0,77 & 0,59 \\
\hline \multirow{6}{*}{$\begin{array}{l}\text { Competencias } \\
\text { intratégicas }\end{array}$} & Comunicación & 3 & 2 & 5 & 4,27 & 0,69 & 0,48 \\
\hline & Coaching & 3 & 2 & 5 & 4,22 & 0,67 & 0,45 \\
\hline & Trabajo en equipo & 3 & 2 & 5 & 4,57 & 0,80 & 0,64 \\
\hline & Dirección de personas & 3 & 2 & 5 & 4,35 & 0,79 & 0,62 \\
\hline & Delegación & 2 & 3 & 5 & 4,19 & 0,74 & 0,55 \\
\hline & Liderazgo & 2 & 3 & 5 & 4,49 & 0,69 & 0,48 \\
\hline \multirow{3}{*}{ Proactividad } & Iniciativa & 3 & 2 & 5 & 4,16 & 0,76 & 0,58 \\
\hline & Creatividad & 3 & 2 & 5 & 4,24 & 0,80 & 0,63 \\
\hline & Optimismo & 2 & 3 & 5 & 4,41 & 0,64 & 0,41 \\
\hline \multirow{3}{*}{$\begin{array}{l}\text { Resolución de } \\
\text { Problema }\end{array}$} & Intuición & 3 & 2 & 5 & 4,35 & 0,89 & 0,79 \\
\hline & Capacidad de análisis & 2 & 3 & 5 & 4,57 & 0,55 & 0,31 \\
\hline & Toma de decisiones & 3 & 2 & 5 & 4,46 & 0,77 & 0,59 \\
\hline \multirow{3}{*}{ Autogobierno } & Concentración & 4 & 1 & 5 & 4,22 & 0,85 & 0,73 \\
\hline & Tenacidad & 3 & 2 & 5 & 4,22 & 0,71 & 0,51 \\
\hline & Autocontrol & 2 & 3 & 5 & 4,14 & 0,63 & 0,40 \\
\hline \multirow{3}{*}{ Gestión personal } & Gestión de tiempo & 2 & 3 & 5 & 4,43 & 0,69 & 0,47 \\
\hline & Gestión de estrés & 3 & 2 & 5 & 4,27 & 0,77 & 0,59 \\
\hline & Gestión de incertidumbre & 3 & 2 & 5 & 4,27 & 0,73 & 0,54 \\
\hline \multirow{3}{*}{ Integridad } & Credibilidad & 2 & 3 & 5 & 4,57 & 0,65 & 0,42 \\
\hline & Honestidad & 2 & 3 & 5 & 4,81 & 0,46 & 0,21 \\
\hline & Equidad & 3 & 2 & 5 & 4,32 & 0,85 & 0,73 \\
\hline \multirow{3}{*}{ Desarrollo personal } & Autocrítica & 3 & 2 & 5 & 4,14 & 0,71 & 0,51 \\
\hline & Autoconocimiento & 2 & 3 & 5 & 4,19 & 0,66 & 0,44 \\
\hline & Aprendizaje personal & 3 & 2 & 5 & 4,27 & 0,80 & 0,65 \\
\hline
\end{tabular}


Figura 2. Importancia de las competencias directivas en las empresas de servicios

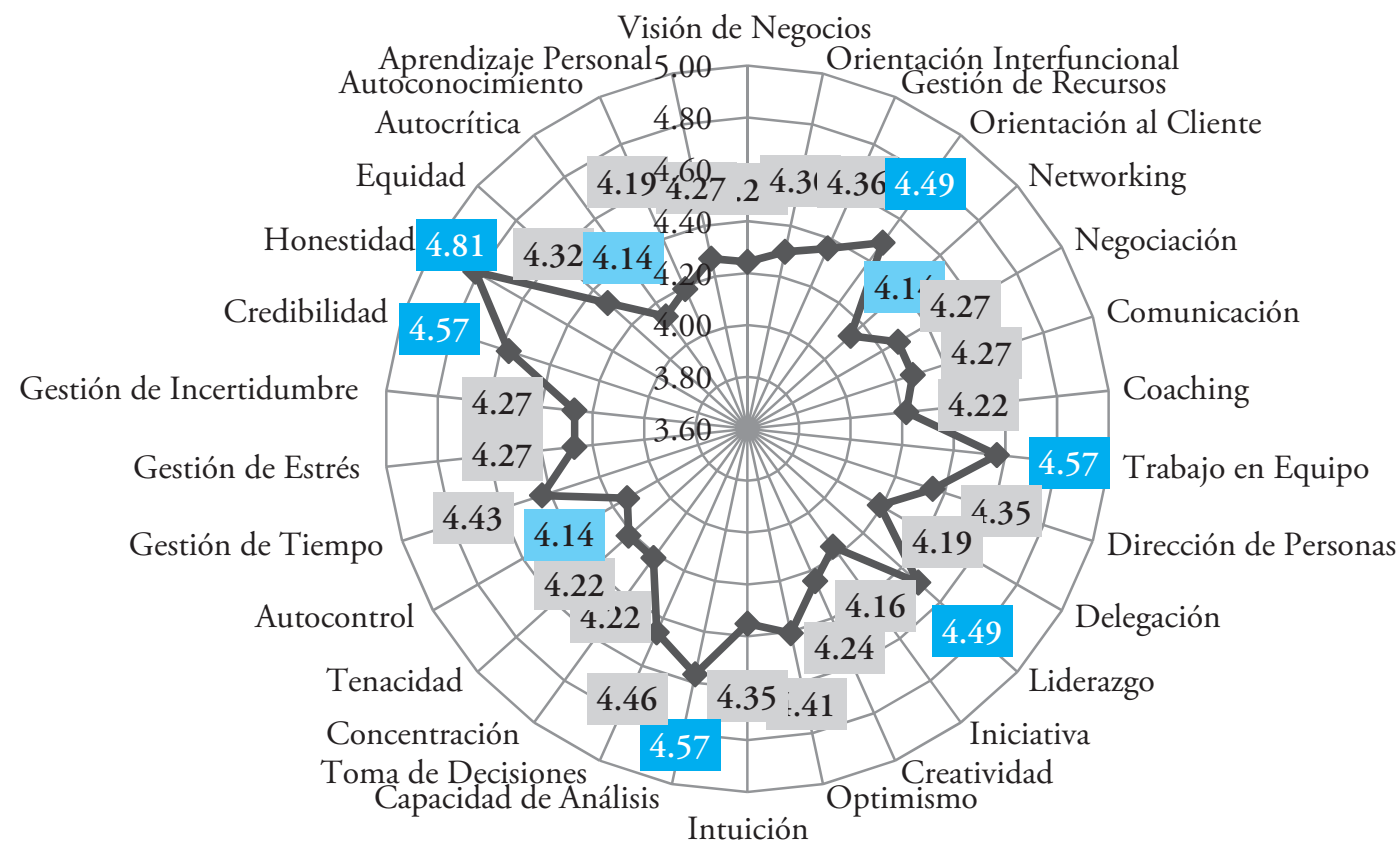

También, se registró una correlación negativa baja entre la edad del evaluador y la competencia intratégica de comunicación $(\mathrm{r}=-, 299 ; \mathrm{p}<, 05)$, lo cual indica que, a mayor edad del evaluador, es menor la importancia otorgada a la competencia comunicación. De igual modo, se observa una correlación negativa moderada entre la edad del evaluador y las competencias directivas: autocontrol $(r=-, 346)$ y honestidad $(r=-, 300)$. Ello evidencia que, a mayor edad del empresario, este asigna menos importancia a las competencias mencionadas.

\subsection{Competencias directivas en las empresas del sector industrial}

Con respecto al rubro de industria-manufactura, se acudió a 18 empresas que conforman el 25\% del total de la muestra, de las cuales casi el $68 \%$ fueron empresas grandes, el 22,22\% fueron medianas empresas y solo 2 empresas fueron pequeñas. En relación con la variable cargo del empresario, casi el $80 \%$ de los entrevistados fueron jefes, uno fue gerente de área y 3 fueron gerentes generales. Con respecto al sexo, 13 empresarios fueron varones - lo que conforma el $72,22 \%$ de esta submuestra-, mientras que solo hubo 5 mujeres —que conformaron el 27,78\% - del total de los 18 entrevistados. En cuanto a la profesión de los empresarios del rubro industrial, el 50\% fueron administradores de negocios, seguidos por las profesiones de ingeniero industrial $(22,22 \%)$ y de contador (16,67\%). También, se evaluó a un economista y a un psicólogo, quienes ocuparon cargos directivos. En cuanto a la edad de los entrevistados, los porcentajes son similares para las 3 categorías de edad, que comprenden de los 30 a los 59 años $(27,78 \%)$. La edad mínima fue de 25 años y la edad máxima de 57 años. El menor porcentaje se concentró entre las edades de 20 a 29 años (16,67\%). 
Tabla 3. Valores descriptivos de la importancia de las competencias directivas en el rubro de industria

\begin{tabular}{|c|c|c|c|c|c|c|c|}
\hline \multirow{7}{*}{$\begin{array}{l}\text { Competencias } \\
\text { estratégicas }\end{array}$} & & Rango & Mínimo & Máximo & Media & D.E. & Varianza \\
\hline & Visión de negocio & 1 & 4 & 5 & 4,67 & 0,49 & 0,24 \\
\hline & Orientación interfuncional & 2 & 3 & 5 & 4,39 & 0,61 & 0,37 \\
\hline & Gestión de recursos & 2 & 3 & 5 & 4,33 & 0,59 & 0,35 \\
\hline & Orientación al cliente & 2 & 3 & 5 & 4,67 & 0,59 & 0,35 \\
\hline & Networking & 2 & 3 & 5 & 4,39 & 0,78 & 0,60 \\
\hline & Negociación & 2 & 3 & 5 & 4,22 & 0,73 & 0,54 \\
\hline \multirow{6}{*}{$\begin{array}{l}\text { Competencias } \\
\text { intartégicas }\end{array}$} & Comunicación & 2 & 3 & 5 & 4,33 & 0,69 & 0,47 \\
\hline & Coaching & 2 & 3 & 5 & 4,06 & 0,42 & 0,17 \\
\hline & Trabajo en equipo & 1 & 4 & 5 & 4,44 & 0,51 & 0,26 \\
\hline & Dirección de personas & 2 & 3 & 5 & 3,94 & 0,64 & 0,41 \\
\hline & Delegación & 3 & 2 & 5 & 4,11 & 0,90 & 0,81 \\
\hline & Liderazgo & 2 & 3 & 5 & 4,22 & 0,65 & 0,42 \\
\hline \multirow{3}{*}{ Proactividad } & Iniciativa & 2 & 3 & 5 & 4,50 & 0,62 & 0,38 \\
\hline & Creatividad & 3 & 2 & 5 & 4,33 & 0,84 & 0,71 \\
\hline & Optimismo & 2 & 3 & 5 & 4,28 & 0,57 & 0,33 \\
\hline \multirow{3}{*}{$\begin{array}{l}\text { Resolución de } \\
\text { problema }\end{array}$} & Intuición & 1 & 4 & 5 & 4,39 & 0,50 & 0,25 \\
\hline & Capacidad de análisis & 2 & 3 & 5 & 4,44 & 0,62 & 0,38 \\
\hline & Toma de decisiones & 2 & 3 & 5 & 4,61 & 0,61 & 0,37 \\
\hline \multirow{3}{*}{ Autogobierno } & Concentración & 2 & 3 & 5 & 4,17 & 0,51 & 0,26 \\
\hline & Tenacidad & 2 & 3 & 5 & 4,28 & 0,57 & 0,33 \\
\hline & Autocontrol & 2 & 3 & 5 & 4,17 & 0,51 & 0,26 \\
\hline \multirow{3}{*}{ Gestión personal } & Gestión de tiempo & 2 & 3 & 5 & 4,56 & 0,62 & 0,38 \\
\hline & Gestión de estrés & 2 & 3 & 5 & 4,33 & 0,59 & 0,35 \\
\hline & Gestión de incertidumbre & 3 & 2 & 5 & 4,00 & 0,77 & 0,59 \\
\hline \multirow{3}{*}{ Integridad } & Credibilidad & 2 & 3 & 5 & 4,50 & 0,62 & 0,38 \\
\hline & Honestidad & 1 & 4 & 5 & 4,72 & 0,46 & 0,21 \\
\hline & Equidad & 1 & 4 & 5 & 4,28 & 0,46 & 0,21 \\
\hline \multirow{3}{*}{ Desarrollo personal } & Autocrítica & 2 & 3 & 5 & 4,06 & 0,54 & 0,29 \\
\hline & Autoconocimiento & 2 & 3 & 5 & 4,06 & 0,54 & 0,29 \\
\hline & Aprendizaje personal & 1 & 4 & 5 & 4,44 & 0,51 & 0,26 \\
\hline
\end{tabular}


Figura 3. Importancia de las competencias directivas en las empresas industriales

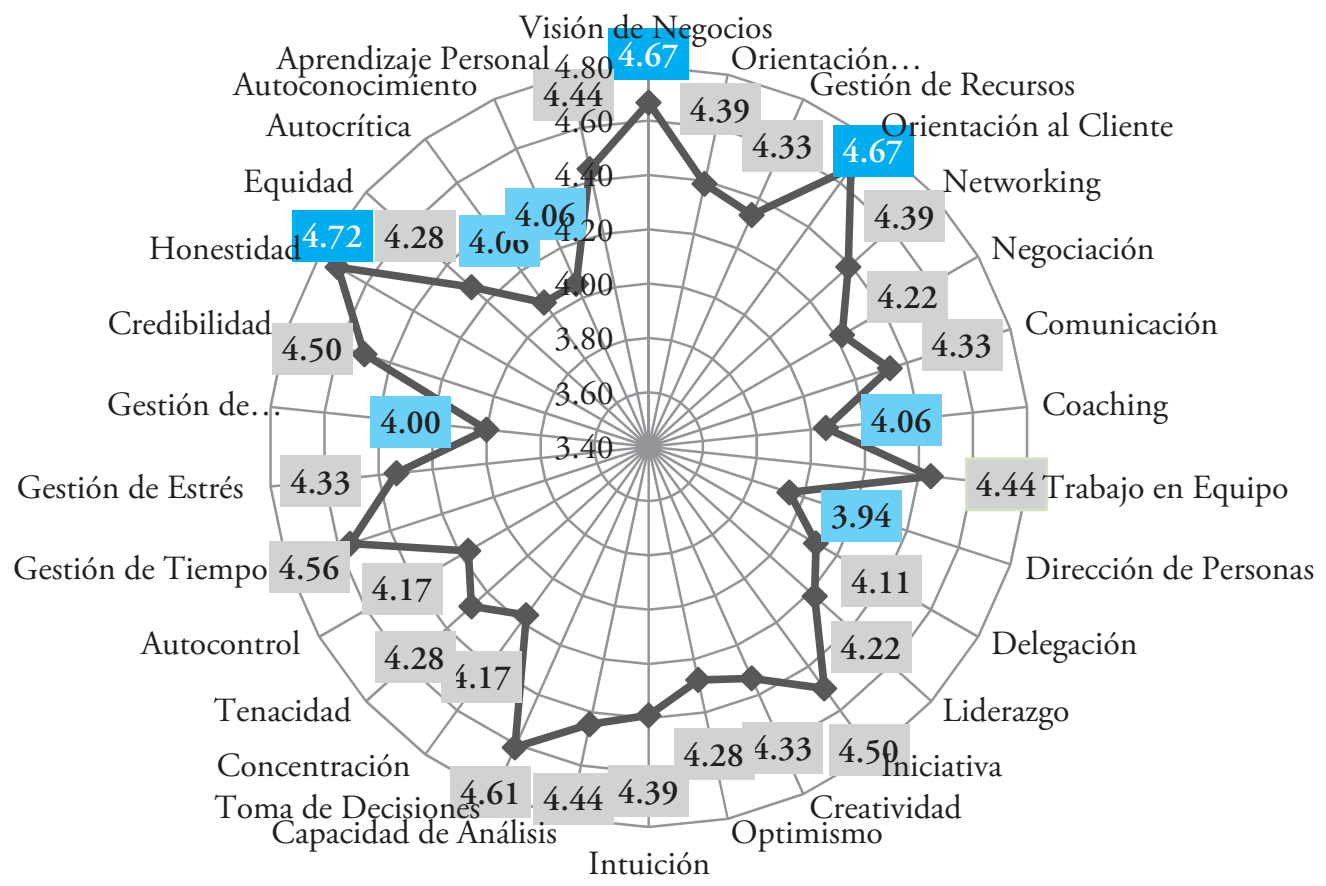

En cuanto a los valores descriptivos de las competencias directivas, las puntuaciones alcanzadas en el rubro industrial también correspondieron a medias altas con desviaciones estándar bajas (Tabla 3). En cuanto a la importancia de las competencias directivas en las empresas industriales, los directivos asignaron puntuaciones altas a la mayoría de las competencias directivas. La competencia que recibió una mayor puntuación promedio fue la competencia de eficacia personal: honestidad. En segundo lugar, están las competencias estratégicas, dentro de las cuales sobresalen la visión de negocios y la orientación al cliente. Por su lado, las competencias que obtuvieron los menores promedios fueron dirección de personas y gestión de incertidumbre, seguidas de autocrítica,

autoconocimiento y coaching. En resumen, las competencias más importantes para los directivos de las empresas industriales son, en su mayoría, las competencias de eficacia personal, seguidas de las competencias estratégicas.

Al practicar las correlaciones con la prueba Tau-b de Kendall, se obtuvo una correlación moderada negativa entre la edad del evaluador, y el trabajo en equipo $(r=-, 441)$, la honestidad $(r=-, 517)$ y la equidad $(\mathrm{r}=-, 499)$ con un nivel de significancia de $\mathrm{p}<, 05$. Dicha correlación da cuenta de que, mientras mayor es la edad del directivo en las empresas industriales, es menor la calificación otorgada a las competencias mencionadas. 


\subsection{Competencias directivas en las empresas del sector comercial}

Con respecto al rubro comercial, se acudió a 15 empresas que conforman el $22 \%$ del total de la muestra. De estas, el $46,67 \%$ estuvo compuesto por pequeñas empresas; el $40 \%$, por grandes empresas; y solo un directivo laboraba en una microempresa y otro directivo, en una mediana empresa. En cuanto al sexo, este subgrupo de directivos está integrado por 9 varones que conforman el $60 \%$ y 5 mujeres que conforman el $40 \%$ del total de los 15 entrevistados. En cuanto al cargo que ostentan, 9 son jefes que conformaron el 60\%; 3 son gerentes generales (20\%); y otros 3, gerentes de área (20\%). Tomando como referente su profesión, nuevamente, la carrera predominante es la de Administración de Negocios (40\%), seguida de Economía y Psicología, ambas con $13,33 \%$. Asimismo, se entrevistó a tres ingenieros, un relacionista industrial y un comunicador. La edad mínima fue de 25 años y la máxima, de 57 años, con un promedio de 39 años de edad. La mayoría de directivos se ubicaron entre los rangos de edades de 30 a 39, y 40 a 49 (ambas con un 33,3\%), lo que fue seguido de un $20 \%$ para las edades de 20 a 29 , mientras que el menor porcentaje lo obtuvieron los directivos cuyas edades se ubican entre 50 y 59 ańos $(13,3 \%)$.

En cuanto a los valores descriptivos, las medias de las competencias directivas fueron medias altas con desviaciones estándar bajas (Tabla 4). Las competencias que recibieron una mayor puntuación promedio fueron las competencias de eficacia personal; específicamente, la honestidad. En segundo lugar, se encuentran equidad y gestión del tiempo. En tercer lugar, están credibilidad, gestión de incertidumbre y creatividad. Por otro lado, las competencias que obtuvieron las menores puntuaciones promedio fueron networking, coaching y autocrítica. Se puede afirmar, entonces, que las competencias más valoradas por los directivos de empresas del sector comercial son las competencias de eficacia personal.

A nivel correlacional (con la prueba Tau-b de Kendall), se encontró una correlación negativa moderada entre el cargo del directivo y las competencias de comunicación $(r=-, 560)$, autocrítica $(r=-598)$ y aprendizaje personal $(r=-560)$, con un nivel de significancia de $\mathrm{p}<, 05$. Ello indica que, mientras mayor sea el cargo del evaluador en las empresas comerciales, este otorga una menor importancia a las competencias mencionadas. También, se halló una correlación negativa alta entre el cargo del evaluador y las competencias de liderazgo $(r=-, 624)$ y creatividad $(r=-, 684)$, con un nivel de significancia de $\mathrm{p}<, 01$. Dicha correlación evidencia que, mientras mayor sea el cargo del evaluador en las empresas comerciales, les asigna menos importancia a las competencias mencionadas y más importancia a aquellas competencias que presentan una correlación moderada.

A diferencia de los otros dos rubros anteriores, en el sector comercial, sí se encontró una correlación positiva alta entre el tamaño de la empresa y la orientación al cliente $(\mathrm{r}=, 647 ; \mathrm{p}<, 05)$, lo cual da cuenta de que, mientras más grande sea la empresa comercial, los ejecutivos entrevistados dan una mayor importancia a la orientación al cliente. Así también, se encontró una correlación positiva, moderada y significativa $(\mathrm{p}<, 05)$ entre el tamaño de la empresa y las siguientes competencias directivas: trabajo en equipo $(\mathrm{r}=, 505)$, liderazgo $(\mathrm{r}=, 520)$, creatividad $(\mathrm{r}=, 581)$, optimismo $(\mathrm{r}=$,557), concentración $(\mathrm{r}=590)$, credibilidad $(\mathrm{r}=, 581)$ y aprendizaje personal $(\mathrm{r}=, 535)$. Estos resultados sugieren que, mientras más grande es la empresa, los ejecutivos entrevistados dan una mayor importancia a las competencias mencionadas. 
Tabla 4. Valores descriptivos de la importancia de las competencias directivas en el rubro comercial

\begin{tabular}{|c|c|c|c|c|c|c|c|}
\hline \multirow{7}{*}{$\begin{array}{l}\text { Competencias } \\
\text { estratégicas }\end{array}$} & & Rango & Mínimo & Máximo & Media & D.E. & Varianza \\
\hline & Visión de negocio & 1 & 4 & 5 & 4,47 & 0,52 & 0,27 \\
\hline & Orientación interfuncional & 2 & 3 & 5 & 4,60 & 0,63 & 0,40 \\
\hline & Gestión de recursos & 2 & 3 & 5 & 4,53 & 0,64 & 0,41 \\
\hline & Orientación al cliente & 1 & 4 & 5 & 4,67 & 0,49 & 0,24 \\
\hline & Networking & 2 & 3 & 5 & 4,13 & 0,64 & 0,41 \\
\hline & Negociación & 2 & 3 & 5 & 4,47 & 0,74 & 0,55 \\
\hline \multirow{6}{*}{$\begin{array}{l}\text { Competencias } \\
\text { intratégicas }\end{array}$} & Comunicación & 2 & 3 & 5 & 4,47 & 0,64 & 0,41 \\
\hline & Coaching & 2 & 3 & 5 & 4,20 & 0,68 & 0,46 \\
\hline & Trabajo en equipo & 2 & 3 & 5 & 4,47 & 0,64 & 0,41 \\
\hline & Dirección de personas & 2 & 3 & 5 & 4,40 & 0,63 & 0,40 \\
\hline & Delegación & 1 & 4 & 5 & 4,40 & 0,51 & 0,26 \\
\hline & Liderazgo & 2 & 3 & 5 & 4,47 & 0,74 & 0,55 \\
\hline \multirow{3}{*}{ Proactividad } & Iniciativa & 3 & 2 & 5 & 4,53 & 0,83 & 0,70 \\
\hline & Creatividad & 1 & 4 & 5 & 4,73 & 0,46 & 0,21 \\
\hline & Optimismo & 2 & 3 & 5 & 4,60 & 0,63 & 0,40 \\
\hline \multirow{3}{*}{$\begin{array}{l}\text { Resolución de } \\
\text { problema }\end{array}$} & Intuición & 3 & 2 & 5 & 4,47 & 0,83 & 0,70 \\
\hline & Capacidad de análisis & 2 & 3 & 5 & 4,47 & 0,74 & 0,55 \\
\hline & Toma de decisiones & 2 & 3 & 5 & 4,60 & 0,63 & 0,40 \\
\hline \multirow{3}{*}{ Autogobierno } & Concentración & 1 & 4 & 5 & 4,60 & 0,51 & 0,26 \\
\hline & Tenacidad & 2 & 3 & 5 & 4,67 & 0,62 & 0,38 \\
\hline & Autocontrol & 3 & 2 & 5 & 4,60 & 0,83 & 0,69 \\
\hline \multirow{3}{*}{ Gestión personal } & Gestión de tiempo & 2 & 3 & 5 & 4,80 & 0,56 & 0,31 \\
\hline & Gestión de estrés & 1 & 4 & 5 & 4,67 & 0,49 & 0,24 \\
\hline & Gestión de incertidumbre & 1 & 4 & 5 & 4,73 & 0,46 & 0,21 \\
\hline \multirow{3}{*}{ Integridad } & Credibilidad & 1 & 4 & 5 & 4,73 & 0,46 & 0,21 \\
\hline & Honestidad & 1 & 4 & 5 & 4,87 & 0,35 & 0,12 \\
\hline & Equidad & 1 & 4 & 5 & 4,80 & 0,41 & 0,17 \\
\hline \multirow{3}{*}{ Desarrollo personal } & Autocrítica & 2 & 3 & 5 & 4,27 & 0,80 & 0,64 \\
\hline & Autoconocimiento & 3 & 2 & 5 & 4,40 & 0,83 & 0,69 \\
\hline & Aprendizaje personal & 2 & 3 & 5 & 4,47 & 0,64 & 0,41 \\
\hline
\end{tabular}


Figura 4. Importancia de las competencias directivas en las empresas industriales

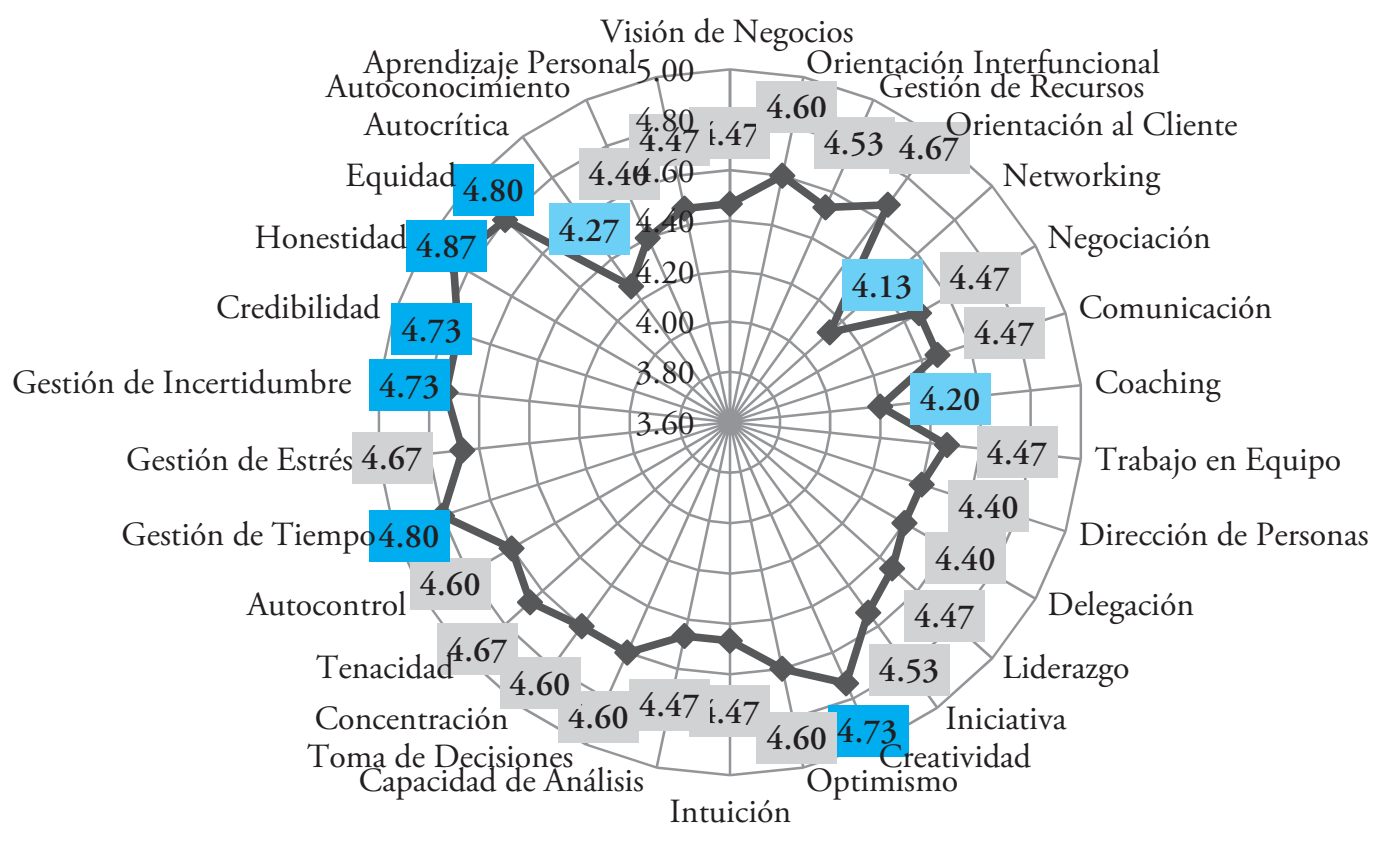

\section{Discusión}

Aunque el enfoque por competencias no es nuevo pues data de los años 50 (White 1959) y se nutre de los modelos comportamentales (Ortiz, Rendón y Atehortúa, 2009)-, hoy en día, tiene una gran difusión y goza de amplia aceptación en el mundo empresarial debido a su fácil aplicación, su utilidad práctica y su operativización objetiva (Alles, 2011). De este modo, las competencias pueden considerarse como indicadores de la conducta o de conductas observables que se presuponen necesarias para el desempeño de un puesto de trabajo (Hamel y Prahalad, 1990). Estas competencias son, entonces, indispensables para la realización de un determinado trabajo, más aún que los conocimientos y la experiencia previa (Mc Clelland, 1973). En este trabajo, se ha tomado en cuenta la división de competencias técnicas y directivas (Cardona et al.,
2001), que responde a un modelo antropológico sobre la base de las ideas de Juan Antonio Pérez López (1993). Dentro de este modelo, las competencias directivas se dividen en tres: estratégicas, intratégicas y de eficacia personal. Estas, a su vez, se alinean coherentemente con ciertos valores, motivos y necesidades, como ya ha sido explicado previamente.

El fin del presente trabajo ha sido dar a conocer la valoración que hacen los empresarios de la ciudad de Arequipa sobre las competencias directivas en tres rubros empresariales, según su importancia. En ese marco, se ha observado que dichas competencias tienen una relevancia moderada para los directivos, pues se les ha asignado una puntuación promedio mayor a 3,94 sobre una puntuación máxima de 5. Además, los directivos de empresas comerciales fueron los que les asignaron las mayores puntuaciones con un promedio 
de 4,54, seguidos por los directivos de las empresas industriales y de servicios, ambas con un promedio de 4,33 .

En ese sentido, las competencias más valoradas fueron visión de negocio en las empresas de servicios; gestión de recursos, en las empresas industriales; y networking, en las empresas comerciales. Estos resultados parecen reflejar el acento en la gestión de los distintos tipos de empresa estudiados, puesto que el análisis de la importancia de cada competencia la vincula a un rubro determinado. Por ejemplo, la gestión de recursos es muy importante en las empresas industriales, pues de esta depende su rentabilidad. De manera similar, en las empresas comerciales, el networking es sumamente importante, en la medida que no existe una empresa comercial que no entable ni necesite de una excelente red de relaciones dentro de la empresa y del sector. Así, como señalan Ortiz et al. (2009), dado que el único factor irrepetible en una empresa es el talento humano, las competencias directivas se han de manifestar de manera muy particular en cada rubro, si no lo hacen también en cada empresa.

Ahora bien, es importante destacar que la orientación interfuncional fue la competencia que estuvo más cerca de haber sido calificada como regular en los tres rubros de empresa. Por ello, es recomendable insistir en la formación de esta competencia. Esta es considerada de alta importancia, debido a que en una organización las áreas no pueden trabajar de forma independiente, pues forman parte de un todo. Además, resulta imposible dirigir una organización si solo se tiene una visión limitada de las demás áreas (Ospina, 2010). A partir de ello, los modelos de enriquecimiento del trabajo proponen que los colaboradores deben tener conocimiento de todo el proceso operativo, ya sea que se trate de la implementación de un servicio o de la elaboración de un producto, lo cual supone cierta interfuncionalidad entre diferentes áreas de la empresa (Schultz, 1999).

Por otro lado, dentro de las competencias intratégicas, el trabajo en equipo fue la competencia que recibió la calificación máxima tanto de empresas industriales como de servicios. En las empresas comerciales, la competencia más valorada fue el liderazgo, pero el trabajo en equipo también es considerado como fundamental con puntuaciones muy similares. En ese sentido, el trabajo en equipo es una variable fundamental del éxito de una empresa que, si bien se desarrolla a partir de la cultura organizacional y de las relaciones interpersonales dentro de la empresa, tiene un impacto profundo en el compromiso organizacional de los trabajadores (Acosta, Salanova y Llorens, 2012) y promueve la empatía colectiva entre ellos (Solares, Peñalver, Meneghel y Salanova, 2016).

En cuanto a las competencias de eficacia personal, los resultados indican que la competencia considerada como más importante en el rubro de servicios fue el autocontrol, mientras que, en las empresas industriales, las competencias más valoradas fueron la concentración, el autocontrol, la equidad, la autocrítica y el autoconocimiento. Por su parte, para los empresarios del rubro comercial, las competencias más importantes fueron la concentración, el autoconocimiento y el aprendizaje personal. Esto supone que los empresarios buscan colaboradores autónomos; proactivos; y con iniciativa, creatividad y autorregulación del aprendizaje. Estas cualidades son fundamentales para el desarrollo organizacional mediado por la gestión de la innovación (Restrepo, 2013).

Finalmente, una competencia muy bien valorada en los tres rubros empresariales fue la honestidad, que forma parte de los valores clave de la ética empresarial (Manzone, 2007), y que, de acuerdo con algunos 
estudios, se encuentra deteriorada entre los administradores y los gerentes (Zerpa y Ramírez 2012). Una competencia que fue valorada como regular en los tres rubros fue el coaching, posiblemente, porque todavía no se ha difundido lo suficiente entre los empresarios arequipeños y aún son escasos los estudios que dan cuenta de sus bondades en el medio local (Sánchez, 2017).

Una utilidad práctica de este estudio es que, a partir de estos datos, se pueden establecer perfiles ocupacionales basados en las competencias. Por ejemplo, en cuanto a las competencias directivas más relevantes, puede decirse que, dadas las competencias seleccionadas en orden de importancia, los directivos de las empresas de servicios estarían en busca de personas honestas, capaces de trabajar en equipo y de realizar análisis para resolver problemas, con credibilidad, que sepan atender las necesidades del cliente, líderes, con capacidad para tomar decisiones y gestionar su tiempo adecuadamente, optimistas, y que sepan dirigir personas.

Por otro lado, los directivos de las empresas industriales estarían en busca de personas honestas; con visión de negocio; que sepan atender las necesidades del cliente y tomar decisiones, y gestionar sus tiempos de forma adecuada; emprendedores; transparentes; capaces de crear equipo, de analizar para resolver problemas, y de aprender de manera flexible. Finalmente, los directivos de las empresas comerciales estarían en busca de personas honestas que sepan gestionar su tiempo, justas, creativas, que tomen decisiones adecuadas en momentos de incertidumbre, transparentes, que sean capaces de responder las necesidades del cliente y tenaces.

Estos perfiles ocupacionales podrían orientar la elaboración de los perfiles de egresados de las universidades locales para que puedan responder de manera más eficiente y precisa a las exigencias laborales del mercado empresarial arequipeño, que constituiría, a su vez, una oportunidad para vincular directamente la universidad con la empresa dentro de un enfoque humanístico (Castrillón y Alzate, 2012). En ese sentido, es necesario ahondar más en las cualidades que requieren los futuros administradores para ejercer su profesión con solvencia académica, profesional y moral. En Lima, por ejemplo, se han investigado los valores organizacionales (Loli y López, 2002) y la capacidad de emprendimiento de los jóvenes universitarios (Loli, Dextre, Del Carpio y La Jara, 2010; Loli, Aliaga, Del Carpio, Vergara y Aliaga, 2011), mientras que en Arequipa se han analizado las relaciones entre las preferencias vocacionales y la identidad profesional de los estudiantes de una escuela de negocios de una universidad privada (Callata, Morales y Arias, 2017). Consideramos que este tipo de estudios permitiría conocer mejor la realidad que van a enfrentar los administradores, y, en esa medida, las casas de estudios superiores pueden tomar medidas en el ámbito formativo que reduzcan la brecha entre lo que se hace y lo que se necesita (Zárate-Hoyos y LariosMeoño, 2015).

Cabe considerar que se tiene un perfil en el que destacan las competencias directivas de eficacia personal y, sobre todo, la honestidad, mientras que, en el estudio original de Cardona et al. (2001), se obtuvo un perfil más equilibrado entre los tres tipos de competencias, con un leve predominio de las competencias intratégicas. Estas diferencias podrían deberse a la distancia temporal que existe entre una y otra investigación, puesto que, a la fecha, los valores de eficacia personal han conseguido penetrar más en el mundo empresarial, en parte, debido al impulso que se les ha dado desde la psicología organizacional positiva (Salanova, Martínez y Llorens, 2005). 
La psicología organizacional positiva se ha centrado en aspectos como el bienestar del trabajador (Marssolier y Aparicio, 2011), los determinantes del compromiso organizacional (Bakker, Demerouti y Xanthopoulou, 2012) y la experimentación de emociones positivas en el trabajo como fuentes de satisfacción laboral (Waters, 2012). En Arequipa, por ejemplo, se han llevado a cabo estudios sobre fenómenos similares como la felicidad, la espiritualidad, la motivación y la gratitud en el trabajo (Arias, Masías, Muñoz y Arpasi, 2013; Menautt y otros, 2016), además de otros trabajos sobre emociones positivas en contextos no organizacionales.

Para concluir, es necesario comentar que también se ha encontrado que los ejecutivos de las empresas de servicios que tienen un mayor cargo asignan menos importancia al trabajo en equipo, la honestidad y el aprendizaje personal. Basándonos en ello, podríamos deducir que los ejecutivos de mayor cargo generalmente dan una mayor importancia a las competencias estratégicas, mientras que, en las empresas comerciales, el tamaño de la empresa se ha relacionado positivamente con la orientación al cliente, el trabajo en equipo, el liderazgo, la creatividad, el optimismo, la concentración, la credibilidad y aprendizaje personal. Estos resultados sugieren que, mientras más grande es la empresa, los empresarios arequipeños dan más importancia a las competencias de eficacia personal. En ese sentido, el tamaño de la empresa no solo tiene un impacto en la estructura organizacional y la productividad, sino también en el comportamiento organizacional, y, por ende, en los estilos directivos y la visión de las competencias necesarias para realizar determinadas funciones dentro de la empresa (Zapata, 2015).

Con este estudio, se ha apuntado a analizar la relevancia de las competencias directivas desde la percepción de los empresarios arequipeños, pues el capital humano tiene una participación vital en la consecución de los fines y metas organizacionales. De hecho, en un estudio realizado en Colombia, se reportó que el capital humano era capaz de explicar el $41 \%$ de la varianza de la rentabilidad empresarial (Zapata, Gutiérrez y Rubio, 2013). En ese sentido, consideramos que es necesario realizar más investigaciones sobre las empresas locales, a través de la aplicación del modelo antropológico de Pérez López, que dé mayor relevancia al capital social y a la gestión del talento humano, puesto que la percepción utilitarista y pragmática del ser humano en la empresa ha cambiado en las últimas décadas (Vélez, 2012). Por otro lado, es importante generar mayor evidencia empírica de la visión antropológica en la empresa, pues no solo se encuentra en sintonía con las tendencias actuales que se focalizan en la persona y los valores humanos, sino que, además, permite responder con eficiencia y eficacia a los problemas organizacionales que acontecen en las empresas de la región.

\section{Referencias}

Acosta, H., Salanova, M. y Llorens, S. (2012). How organizational practices predict team work engagement: The role of organizational trust. Ciencia \& Trabajo, 14, 7-15.

Alles, M. (2010). Dirección estratégica de recursos humanos. Gestión por competencias. Barcelona: Granica.

Alles, M. (2011). Desempeño por competencias. Evaluación de $360^{\circ}$. Barcelona: Granica.

Alles, M. (2012). Selección por competencias. Barcelona: Granica.

Arias, W. L., Masías, M. A., Muñoz, E. y Arpasi, M. (2013). Espiritualidad en el ambiente laboral y su relación con la felicidad del trabajador. Revista de Investigación de la Universidad Católica San Pablo, 4, 9-33.

Contabilidad y Negocios (13) 26, 2018 / ISSN 1992-1896 
Bakker, A., Demerouti, E. y Xanthopoulou, D. (2012). How do engaged employees stay engaged? Ciencia \& Trabajo, 14, 16-22.

Barroso, F. G. (2008). La responsabilidad social empresarial. Un estudio en cuarenta empresas de la ciudad de Mérida, Yucatán. Contaduría y Administración, 226, 73-91.

Burga, A. (2006). La unidimensionalidad de un instrumento de medición: perspectiva factorial. Revista de Psicología de la Pontificia Universidad Católica del Perú, 24(1), 53-80.

Callata, M., Morales, A. y Arias, W. L. (2017). Identidad profesional y preferencias profesionales en estudiante de la Escuela Profesional de Administración de Negocios de una universidad privada de Arequipa. Revista de Investigación en Psicología, 20(1), 147-176. https:// doi.org/10.15381/rinvp.v20i1.13529

Cardona, P., Chinchilla, N. y García-Lombardía, P. (2001). IESE insight Business Knowledge Portal. IESE Business School. University of Navarra. Recuperado el 15 de mayo de 2013, de http://www.iese.edu/research/pdfs/ op-01-04.pdf

Castrillón, L. A. y Alzate, G. M. (2012). Humanismo, universidad y empresa: la RSE desde la perspectiva ética. Revista de Ciencias Estratégicas, 20, 27, 171-184.

Chávez, L. L. (2013). Competencias directivas del alumno y egresado del Programa Profesional de Administración de Negocios de la Universidad Católica San Pablo: La percepción de los empresarios arequipeños. Tesis de licenciatura en Administración de Negocios. Arequipa: Universidad Católica San Pablo, Escuela Profesional de Administración de Negocios.

Chiavenato, I. (2002). Administración de recursos humanos. México D.F.: McGraw-Hill.

Chiavenato, I. (2009). Gestión del talento humano. México D.F.: McGraw-Hill.
Covey, S. (1993). Los siete hábitos de la gente altamente efectiva. Barcelona: Paidós.

Drucker, P. (2003). El Management del futuro. Buenos Aires: Editorial Sudamericana.

Fayol, H. (1973). Administración industrial y general. Buenos Aires: El Ateneo.

Ferreiro, P. y Alcázar, M. (2002). Gobierno de personas en la empresa. Lima: Universidad de Piura.

Fischman, D. (2010). La alta rentabilidad de la felicidad. Lima: Universidad Peruana de Ciencias Aplicadas.

Friedman, M. y Friedman, R. (1986). La libertad de elegir. Madrid: Orbis, S. A.

Hamel, G. y Prahalad, C. (1990). The core competence of the corporation. Harvard Business Review, 90(3), 79-91.

Hernández, R., Fernández, C. y Baptista, P. (2010). Metodología de la Investigación. Quinta edición. México D.F.: Mc-Graw Hill.

Jolis, N. (1998). Compétences et compétitivité. París: Les éditions d'organisation.

Loli, A. y López, E. (2002). La autoestima y los valores organizacionales en estudiantes universitarios y no estudiantes universitarios de Lima. Revista de Investigación en Psicología, 5(1), 141-155. https://doi. org/10.15381/rinvp.v20i1.13529

Loli, A., Dextre, E., Del Carpio, J. y La Jara, E. (2010). Actitudes de creatividad y emprendimiento en estudiantes de la Universidad nacional de Ingeniería y su relación con algunas variables sociodemográficas. Revista de Investigación en Psicología, 13(2), 139-151. https://doi.org/10.15381/rinvp.v13i2.3722

Loli, A., Aliaga, J., Del Carpio, J., Vergara, A. y Aliaga, R. (2011). Actitudes de creatividad y emprendimiento y la intención de desarrollar un negocio en estudiantes de la Universidad Nacional Agraria-La Molina. Revista de Investigación en Psicología, 14(1), 209-234. https://doi.org/10.15381/rinvp.v14i1.2084 
Loza, C. y Habisch, A. (2009). Empresa, empresario y consumidor al servicio del desarrollo humano integral según la Caritas in Veritate. Persona y Cultura, 7(7), 22-32.

Manzone, G. (2007). La responsabilidad de la empresa. Business Ethics y la doctrina social de la Iglesia en diálogo. Arequipa: Universidad Católica San Pablo.

Marssolier, R. y Aparicio, M. (2011). El bienestar psicológico en el trabajo y su vinculación con el afrontamiento en situaciones conflictivas. Psicoperspectivas, 10(1), 209-220. https://doi.org/10.5027/psicoperspectivasVol10-Issue1-fulltext-125

Mc Clelland, D. (1973). Testing for competence rather than for intelligence. American Psychologist, 28(1), 1-14. https://doi.org/10.1037/h0034092

Menautt, F., Checa, S. y Arias, W. L. (2016). La gratitud y la percepción de equidad en el trabajo como predictores de la motivación laboral en una agencia bancaria. Revista de Psicología de la Universidad Católica San Pablo, 6(1), 67-90.

Montoya, C. A. (2010). Crisis financiera y económica mundial a la luz de la encíclica «La Caridad en la Verdad». Revista Ciencias Estratégicas, 18(23), 111-118.

Ortiz, J. E., Rendón, M. y Atehortúa, J. (2009). Sobre la necesidad de un nuevo enfoque para la identificación, medición y desarrollo de competencias. Revista Ciencias Estratégicas, 17, 22, 243-250.

Ospina, H. (2010). Nuevos paradigmas en gestión humana. Revista Ciencias Estratégicas. 18(23), 79-96.

Pérez López, J. A. (1993). Fundamentos de la dirección de empresas. Madrid: Rialp.

Quiñones, C. (2013). Human Age: La era de las personas y el talento. Girona: Manpower Group Solutions.

Restrepo, C. A. (2013). Aproximación a la gestión de la innovación: Un enfoque conceptual. Revista Ciencias Estratégicas, 22(30), 257-266.
Robbins, S. (1999). Comportamiento organizacional. Décima edición. México D.F.: Pearson Educación.

Rodríguez, R. L., Martínez, M. y Salanova, M. (2014). Justicia organizacional, engagement en el trabajo y comportamiento de ciudadanía organizacional: Una combinación ganadora. Universitas Psychologica, 13(3), 961-974. https://doi.org/10.11144/Javeriana. UPSY13-3.joet

Salanova, M. (2008). Organizaciones saludables: Una aproximación desde la psicología positiva. En C. Vázquez y G. Hervás (eds.), Psicología positiva aplicada (pp. 403-427). Madrid: Desclée de Brouwer.

Salanova, M., Martínez, I. y Llorens, S. (2005). Psicología organizacional positiva. En F. J. Palací (coord.), Psicología de la organización (pp. 349-376). Madrid: Pearson Educación.

Sánchez, M. (2017). Taller de coaching para mejorar la satisfacción de las relaciones interpersonales en una institución educativa de Arequipa. Illustro, 8, 33-52.

Schultz, D. (1999). Psicología industrial. Bogotá: McGrawHill.

Solares, J., Peñalver, J., Meneghel, I. y Salanova, M. (2016). Desarrollo de equipos empáticos: la influencia de las prácticas organizacionales en la empatía colectiva. Revista de Psicología de la Universidad Católica San Pablo, 6(1), 51-63.

Taylor, F. W. (1973). Principios de la administración cientifica. Buenos Aires: El Ateneo.

Vélez, T. (2012). A theoretical reflection on how human beings have been viewed as workers, from academic and pragmatic perceptions, since the beginning of the twentieth century. Revista Ciencias Estratégicas, 20(27), 161-170.

Waters, L. (2012). Predicting job satisfaction: Contributions of individual gratitude and institutionalized gratitude. Psychology, 3, 1174-1176. https://doi.org/10.4236/ psych.2012.312A173

Contabilidad y Negocios (13) 26, 2018 / ISSN 1992-1896 
White, R. (1959). Motivation reconsidered: The concept of competence. American Psychologist, 66(5), 297-333.

Yamada, G., Castro, J. F. y Asmat, R. (2013). Inversión en educación e ingresos laborales. Lima: Universidad del Pacífico.

Zapata, G. (2015). El tamaño y la estructura de la organización. Un estudio teórico y empírico con el enfoque contingente. Revista Ciencias Estratégicas, 23(34), 193-208.

Zapata, J. C., Gutiérrez, S. y Rubio, M. (2013). El rol del capital humano en la generación de valor: Variables determinantes. Revista Ciencias Estratégicas, 21(29), $31-47$.
Zárate-Hoyos, G. y Larios-Meońo, F. (2015). The role of universities and other institutions in successful entrepreneurship: Some insights from a literature review. Propósitos y Representaciones, 3(2), 261-287.

Zerpa, C. E. y Ramírez, J. (2012). Desarrollo moral en estudiantes de postgrados de gerencia: una comparación. Visión Gerencial, 11(2), 427-445.

Fecha de recepción: 01 de junio de 2018 Fecha de aceptación: 28 de agosto de 2018 Correspondencia: laura.chavez@ucsp.edu.pe warias@ucsp.edu.pe 